\title{
Spike-Directed Vaccination Elicits Robust Spike- Specific T cell Responses Including to Mutant Strains
}

\section{Maja Stanojevic}

Children's National Hospital

\section{Ashley Geiger}

Children's National Hospital

\section{Brita Ostermeier}

Children's National Hospital

\section{Danielle Sohai}

Children's National Hospital

Christopher Lazarski

Children's National Hospital

\section{Haili Lang}

Children's National Hospital

\section{Mariah Jensen-Wachspress}

Children's National Hospital

\section{Kathleen Webber}

Children's National Hospital

\section{Peter Burbelo}

National Institute of Dental and Craniofacial Research

\section{Jeffrey Cohen}

National Institute of Allergy and Infectious Disease

\section{Michael Keller}

Children's National Hospital

\section{Catherine Bollard}

Children's National Hospital

Conrad Russell Cruz ( $\sim$ cCruz@childrensnational.org)

Children's National Hospital

\section{Article}

Keywords: SARS-CoV-2, T cell responses to coronavirus, vaccination, COVID-19 variants 
Posted Date: April 19th, 2021

DOl: https://doi.org/10.21203/rs.3.rs-403449/v1

License: (c) (i) This work is licensed under a Creative Commons Attribution 4.0 International License. Read Full License

Version of Record: A version of this preprint was published at Cytotherapy on September 1st, 2021. See the published version at https://doi.org/10.1016/j.jcyt.2021.07.006. 


\section{Spike-Directed Vaccination Elicits Robust Spike-Specific T cell Responses Including to Mutant Strains}

Maja Stanojevic, ${ }^{*, 1}$ Ashley Geiger, ${ }^{1}$ Brita Ostermeier, ${ }^{2}$ Danielle Sohai, ${ }^{1}$ Christopher Lazarski, ${ }^{1}$ Haili Lang, ${ }^{1}$ Mariah Jensen-Wachspress, ${ }^{1}$ Kathleen Webber, ${ }^{1}$ Peter Burbelo, ${ }^{3}$ Jeffrey Cohen, ${ }^{4}$ Michael D. Keller, ${ }^{1}$ Catherine M. Bollard ${ }^{* 1}$ and Conrad Russell Y. Cruz ${ }^{\star, 1}$

${ }^{1}$ Program for Cell Enhancement and Technologies for Immunotherapy, Children's National Medical Center, Washington DC

${ }^{2}$ Institute of Biomedical Sciences, George Washington University, Washington DC

${ }^{3}$ National Institute of Dental and Craniofacial Research, National Institutes of Health, Bethesda, $\mathrm{MD}$

${ }^{4}$ National Institute of Allergy and Infectious Disease, National Institutes of Health, Bethesda, MD

*These authors contributed equally to this manuscript

Correspondence should be addressed to:

Catherine Bollard, M.D., FRACP, FRCPA

and

C. Russell Y. Cruz MD PhD

Children's National Hospital

Children's National Hospital

5th FIr, Rm. M5225

$5^{\text {th }}$ FIr, Fm M5347

111 Michigan Ave NW

111 Michigan Ave NW

Washington, DC 20010

Washington, DC 20010

CBollard@childrensnational.org

ccruz@childrensnational.org

(202)476-4776

(202)476-2046 


\section{KEYWORDS}

SARS-CoV-2, T cell responses to coronavirus, vaccination, COVID-19 variants 
While most studies describing COVID-19 vaccine responses have focused on antibodies, there is increasing evidence that $T$ cells play a critical role. Here we evaluated $T$ cell responses in seronegative donors before and after vaccination to define responses to SARS-CoV-2 reference strain, as well as to mutations in the variant strains B.1.1.7 and B.1.351. We observed enhanced $T$ cell responses to reference and variant Spike strains post vaccination.

The B.1.1.7 (UK) ${ }^{1}$ and B.1.351 (South African) $)^{2}$ strains, with multiple mutations in the Spike protein (the structural protein serving as antigen for almost all COVID-19 vaccines), have been spreading globally. These two mutant strains are among the variants of concern spreading across the United States, with 5567 reported cases of B.1.1.7 across 50 jurisdictions, and 180 cases of B.1.351 across 26 jurisdictions. ${ }^{3}$ The mutations within the Spike protein are within the receptor binding domain, which influences virus attachment and entry into cells - with some studies suggesting increased binding affinity, and consequently, increased infectivity and transmission. ${ }^{4}$ Recently, different studies have suggested that there is diminished neutralization of the two strains even after vaccination: ${ }^{5}$ sera from vaccinated individuals had neutralization titers several logs lower than those of the reference strain. ${ }^{6,7}$ This raises appreciable concerns regarding the efficacy of current vaccine strategies to control the pandemic.

T cell responses are a critical complementary immune response to antibody responses. Indeed, in individuals with combined variable immune deficiency, rates of COVID-19 hospitalizations and mortality parallel those of the general population, suggesting that in these B cell deficient individuals, T cell response plays a key immunologic role for viral control. ${ }^{8}$ Previous studies have already determined the ability of T cells derived from convalescent individuals to recognize SARSCoV-2, ${ }^{9}$ and presence of virus-specific T cells correlates with protection from severe COVID19and with disease severity and recovery. ${ }^{10}$ 
However, while antibody responses have been well characterized in individuals who have received most of the approved vaccines, ${ }^{6,7,11-13}$ there is currently a paucity of data available on the breadth and cross-reactivity of the T cell responses to mutant viral strains post vaccination. A study by Tarke et al $^{14}$ suggests that variants have a negligible impact on responses post vaccination; however, the antibody and $\mathrm{T}$ cell status of these donors prior to vaccination are unknown, and it is unclear whether prior asymptomatic infections contributed to this post vaccine response.

Hence, we sought to define vaccine specific T cell responses in documented seronegative donors who had no prior history of SARS-CoV-2 and evaluated their response to SARS-CoV-2 structural proteins pre- versus post-vaccination. This would allow interpretation of responses to be assigned solely to the vaccine, and not pre-existing immunity following COVID-19 infection. We investigated the T cell response in seronegative donors with no history of SARS-CoV2 infection. We evaluated T cells derived from six donors who had no detectable antibody responses (NIH) to Spike and Nucleocapsid (Figure 1A, median IgG antibody levels of 35094 light units for nucleocapsid, and 9569 for Spike, $\mathrm{n}=6$; all below seropositive threshold of 125,000 and 45000 light units, respectively) immediately prior to vaccination. All donors also had documented absence of COVID-19 infection by antibody testing in the months before vaccination became available (Supplemental Figure 1). For five of six donors, there was also documented negative COVID$19 \mathrm{PCR}$ /antigen testing (with three of the six donors showing negative results within the week preceding the blood draw obtained immediately before vaccination \#1.

Amplification of specific responses by culturing PBMC in cytokines and antigen for 10 days can be used to enhance sensitivity of determining immune responses. ${ }^{15}$ Hence, we evaluated SARSCoV-2 specific $\mathrm{T}$ cell responses in the peripheral blood immediately after blood collection (D0) 
and after a 10-day expansion step to amplify the response (Supplemental Figure 1). With this approach, we demonstrate reliable amplification post vaccination, similar to what we have observed in antigen-exposed, convalescent donors. ${ }^{15}$ However, even after this expansion step, no detectable responses were observed in our pre-vaccine donor cohort (Figure 1B, Supplemental Figure 2). In contrast, after two doses of vaccine, increased T cell responses to Spike were observed in all donors (Figure 1B, Supplemental Figure 2). Of note, Spike responses were reliably detectable after the second vaccination, in contrast to antibody responses which were detected after the first dose (Figure 1A). ${ }^{16}$

Because current circulating SARS-CoV-2 variants threaten to prolong the pandemic, and observations of decreased neutralization activity from antibodies have been made, ${ }^{6,12}$ we next evaluated potential Spike T cell responses to the mutated regions in currently circulating major variants in the United States: B.1.1.7 and B.1.351 in our post vaccine donor cohort. Epitope predictions of both class I and class II binding suggest the potential for cross reactivity since epitopes from the variant are predicted to bind to the same HLA alleles as their respective reference peptides (Supplemental Tables 2-3) in our donor pool. As shown in Supplemental Tables 2-3, some variant epitopes are predicted to be even stronger binders than the reference homologous epitopes. Regions of the mutations in variants are also a rich source of other HLAbinding epitopes not present in the reference strain (Supplemental Tables 4-5).

We confirmed the potential cross-reactivity of the post vaccine $T$ cell responses following observations that $\mathrm{T}$ cell products stimulated with the reference Spike (Supplemental Table 6) were also able to recognize mutated peptides in the B.1.1.7 variant and additional mutated peptides in the the B.1.351 variant in all but one donor (Figure 2A, Supplemental Figure 3), which is in contrast to the variable antibody responses reported in the literature. ${ }^{5-7,12,17}$ To verify cross-reactivity, we next sought to amplify the response by specifically expanding $\mathrm{T}$ cells using 
the non-redundant mutated sequences within the B.1.1.7 and B.1.351 variant Spike peptide libraries (Supplemental Table 7) as stimulating antigens, and determined responses against reference Spike peptides. In B.1.1.7 Spike-stimulated T cells, we observed reactivity to the variant Spike in 5 out of 6 donors (Figure 2B, Supplemental Figure 4). In B.1.351 Spike-stimulated T cells, we observed reactivity to the variant Spike in 5 out of 6 donors (Figure 2C, Supplemental Figure 5). These observations strongly suggest that epitope-specific $\mathrm{T}$ cell responses to reference Spike cross-react with epitopes in the mutated regions of the B.1.1.7 and B.1.351, consistent with homologies in these predicted epitopes (Supplemental Figure 1) for the HLA types represented by the seronegative donors we evaluated (Supplemental Table 2).

To determine whether CD4+ T cells or CD8+ T cells contribute to the reactivity to Spike, we performed intracellular cytokine stains, and showed that both CD4 and CD8 compartments show specificity against reference Spike protein following vaccination (Figure 2D, 2E, Supplemental Figure 6). CD4+ and CD8+ T cells secreted IFN-y and TNF- $\alpha$ in response to the Spike antigen, and in some donors, also recognize Spike from B.1.1.7 or B.1.351.

This data supports the hypothesis that COVID-19 vaccination elicits immunological responses that are cross-reactive with novel strains, and may be protective through a combination of humoral and adaptive $T$ cell immunity. This data also suggests that immunity may be incomplete after the first vaccine dose. Cellular immune responses peaked after the second Spike mRNA vaccine dose, emphasizing the importance of completing the validated 2-dose vaccine strategy advocated by the manufacturers to elicit both humoral and cell-mediated immunity to SARS-CoV-2 in vivo. The presence of CD8+ T cell responses in donors post vaccination is therefore encouraging since cytotoxic $T$ cells directly lyse their targets, and we posit that their presence may offset the unwanted excessive cytokine secretion mediated by unregulated T cell activity. 
In conclusion, we contend that ongoing surveillance of both antibody and $\mathrm{T}$ cell responses to emerging variants is an important measure to evaluate the immune protection afforded by current mRNA vaccines. T cell response data can potentially serve as indicators for future vaccination strategies including the need for booster vaccines, as well as designs for more global, universal vaccines.

\section{METHODS}

\section{Donors}

Blood was obtained from seronegative donors with no history of SARS-CoV2 infection after obtaining informed consent approved by the IRB. Age, gender and ethnicity of the donors are shown in Supplemental Table 1. All donors had a documented absence of COVID-19 infection by antibody testing $(\mathrm{NIH})$ immediately prior to the blood draw (Figure 1A) and in the months before vaccination became available (Supplemental Figure 1). Peripheral blood mononuclear cells (PBMCs) obtained prior to and after vaccination with each dose of the Pfizer BNT162b2 mRNA vaccine were isolated by density gradient centrifugation using Lymphoprep (STEMCELL Technologies, Cambridge, MA). HLA typing of the healthy donor PBMCs was performed by Kashi Clinical Laboratories, Portland, OR or The Sequencing Center, Fort Collins, CO.

\section{SARS-CoV-2 Peptides}

SARS-CoV-2 structural peptide libraries consisting of 15-mer peptides with overlapping by 11 amino acids and spanning the entire sequence of viral structural proteins: nucleocapsid, Spike, envelope and membrane were designed from the SARS-CoV-2 reference sequence (NC_045512.2) (A\&A peptide, San Diego, CA, USA), mutated regions in the B.1.1.7 variant (TC Peptide Lab peptide, San Diego, CA, USA), and additional mutated sequences within the 
B.1.1351 variant (TC Peptide Lab peptide, San Diego, CA, USA), to identify epitopes. All peptides were reconstituted at working concentration of $1 \mu \mathrm{g} / \mu \mathrm{l}$ in DMSO and stored at $-80^{\circ} \mathrm{C}$ until further use.

\section{Antibody Testing}

Antibodies were determined as previously described. ${ }^{18}$ Plasma was obtained from samples up to 24 hours following blood draw by centrifugation at $1000 \mathrm{G}$ for 15 minutes. Samples were incubated with spike and nucleocapsid proteins fused to Gaussia and Renilla luciferase enzymes. Luciferase activity was measured in light units with a Berthold 165 LB 960 Centro Microplate Luminometer.

\section{Expansion of SARS-CoV-2 T Cells}

In Figure 1, peripheral blood mononuclear cells (PBMCs) were pulsed with an overlapping peptide mix (as described in Keller et $\mathrm{al}^{15}$ ) of viral structural proteins: Nucleocapsid, Spike, Envelope and Membrane. In Figure 2, PBMCs were separately pulsed with a mix of (1) overlapping peptides spanning the reference Spike (Supplemental Table 6) , (2) peptides spanning mutated sequences in Spike variant B.1.1.7 (B.1.1.7 Variant Column on Supplemental Table 7), and (3) the additional, non-redundant unique peptides spanning mutated sequences in Spike variant B.1.351 (B.1.351 Variant Column on Supplemental Table 7); cells were then resuspended with IL-15 (5 ng/mL; R\&D Systems, Minneapolis, MN) and IL-7 (10 ng/ml; R\&D Systems) in CTL media consisting of Click's medium (Irvine Scientific, Santa Ana, CA), RPMI (GE Healthcare, Logan, UT), and human AB serum (Gemini BioProducts, West Sacramento, CA), and supplemented with GlutaMax (Gibco, Grand Island, NY), as previously described. ${ }^{15}$ Cells were fed regularly and split when confluent. Cells were harvested on day ten or eleven and evaluated for antigen specificity by ELISpot. 


\section{Anti-IFN-y enzyme-linked immunospot (ELISpot) assay}

IFN-y enzyme-linked immunospot (ELISpot) assay was performed as previously described, with some modifications. ${ }^{15}$ Antigen specificity of expanded T cells from PBMC grown in the presence of SARS-CoV-2 pepmixes were evaluated by stimulating expanded cells with SARS-CoV-2 pepmixes ${ }^{15}$ and measuring IFN-y production by ELISpot assay. T cells were plated at $1 \times 10^{5}$ cells/well with no peptide, actin PepMix (negative control), Phytohemagglutinin (PHA/positive control), and each of the individual SARS-CoV-2 pepmixes ( $1 \mu \mathrm{g} /$ peptide/well). Antigen specificity of expanded T cells from PBMC grown in the presence of peptides spanning Spike (Supplemental Table 6), peptides spanning B.1.1.7 mutated proteins (B.1.1.7 Variant Column on Supplemental Table 7), or peptides spanning additional B.1.351 mutated proteins (B.1.351 Variant Column on Supplemental Table 7) - with no overlapping sequences between the three - were evaluated by stimulating expanded cells with Spike, B.1.1.7, or B.1.351 pepmixes ${ }^{15}$ and measuring IFN-y production by ELISpot assay. T cells were plated at $1 \times 10^{5}$ cells/well with no peptide, actin PepMix (negative control), Phytohemagglutinin (PHA/positive control), and each of the individual Spike/variant pepmixes ( $1 \mu \mathrm{g} /$ peptide/well). Spot-forming cells (SFCs) were independently counted by Zellnet Consulting (Fort Lee, NJ). Responses that were at least 10 SFC $/ 1 \times 10^{5} \mathrm{~T}$ cells and greater than two-fold the background level of nonstimulated $\mathrm{T}$ cells or $\mathrm{T}$ cells stimulated with actin were considered positive responses.

\section{Intracellular Cytokine Stain}

Ex vivo expanded $T$ cells were frozen after 10 days culture, and thawed the day before intracellular cytokine staining in the presence of $40 \mathrm{U} / \mathrm{mL}$ IL2. Intracellular cytokine stain was performed as previously described, with some modifications. ${ }^{15}$ Briefly, T cells were stimulated with either actin (negative control), Spike peptides, or PHA (positive control) at a concentration of $1 \mu \mathrm{g} / \mathrm{mL}$, in the presence of CD28/49d antibody (BD Fastlmmune) and brefeldin A (BD 
Fastlmmune), and incubated at $37^{\circ} \mathrm{C} 5 \% \mathrm{CO} 2$. After 6 hours, T cells were then washed in $2 \%$ FBS phosphate-buffered saline and surface stained with fluorochrome-conjugated antibodies to CD3, CD4, and CD8. Cells were then fixed, permeabilized using BD Cytofix/Cytoperm solution, and stained with anti-IFN- $y$ and anti-TNF- $\alpha$. T cells were fixed until acquired the following day and then were analyzed on a BD Cytoflex, with data analysis performed using FlowJo (FlowJo LLC, Ashland, OR).

\section{Epitope Analysis}

Predicted HLA Class I were derived from artificial neural network prediction by NetMHCpan 4.1. ${ }^{19}$ Sequences were inputted with no predetermined peptide length selection, alleles of the recruited donors (where no specific type was available, the HLA supertype representative was used), and with a threshold of $0.5 \%$ rank for strong binders. Predicted HLA Class II epitopes were derived from the Immune Epitope Database and Analysis Resource. ${ }^{20}$ Sequences were inputted as peptides (listed in Supplemental Table 7) with the IEDB recommended 2.22 prediction method, selecting for the full HLA reference set with select alpha and beta chains analyzed separately where applicable. Percentile ranks $<2$ were considered strong binders, and percentile ranks between 2-10 were considered weak binders.

\section{AUTHOR CONTRIBUTIONS}

H.L. and M.D.K. designed peptide libraries. H.L. and M.J.W. prepared peptide libraries. K.W. consented donors and obtained blood samples. P.B. and J.C. measured antibody from plasma. M.S., A.G., D.S., and B.O. expanded T cells and performed ELISpot. M.S., C.L., and C.R.Y.C. performed intracellular cytokine staining. M.S. and C.R.Y.C. performed epitope predictions. M.S., M.D.K., C.M.B., and C.R.Y.C. conceived the study, designed the experiments, analyzed the data, and wrote the manuscript. All authors reviewed and edited the manuscript prior to submission. 


\section{ACKNOWLEDGMENTS}

This work was partially supported by the intramural research program of the National Institute of Allergy and Infectious Diseases (NIAID) and the National Institute of Dental and Craniofacial Research (NIDCR).

\section{CONFLICT OF INTEREST}

C.L., M.D.K., C.R.Y.C. and C.M.B. have intellectual property related to developing T cell therapies for infectious disease, including against SARS-CoV-2. All other authors declare no competing interests

\section{FIGURE LEGENDS}

Figure 1. Antibodies and $T$ cells from seronegative donors demonstrate enhanced reactivity to Spike post vaccination. A. Antibody to nucleocapsid and to Spike, measured in six donors immediately before vaccination (blue circles), three weeks after the first vaccine and before administration of the second vaccine (red circles), and three to four weeks after administration of the second vaccine (green circles). B. T cell responses as measured by IFN- $\gamma$ ELISpot after 10-11 day expansion of peripheral blood mononuclear cells with COVID-19 nonstructural antigens Spike, membrane, nucleocapsid, and envelope to zoom in on specific T cells, measured immediately before vaccination (blue circles), three weeks after the first vaccine and before administration of the second vaccine (red circles), and three to four weeks after administration of the second vaccine (green circles). 
Figure 2. T cells exhibit cross-reactivity against B.1.1.7 and B.1.351 variants. A. T cell responses as measured by IFN-ץ ELISpot after 10-11 day expansion of peripheral blood mononuclear cells (PBMCs) with COVID-19 reference Spike. Bars depict mean, and error bars show standard deviation. B. T cell responses as measured by IFN-y ELISpot after 10-11 day expansion of peripheral blood mononuclear cells with COVID-19 B.1.1.7 Spike variant. Bars depict mean, and error bars show standard deviation. C. T cell responses as measured by IFN-y ELISpot after 10-11 day expansion of peripheral blood mononuclear cells with COVID-19 reference Spike. Bars depict mean, and error bars show standard deviation. D. Representative intracellular stain of a donor, showing responses to negative control (actin), to positive control (PHA), and to the three different Spike sequences: reference Spike containing the complete sequence of reference peptides for the Spike protein, B.1.1.7 Spike containing the mutated sequences present in the B.1.1.7 variant that differs from the reference strain, and B.1.351 Spike containing the mutated sequences present in the B.1.351 variant that differs from reference. E. A summary of responses, measured as percent of CD4+ or CD8+ T cells that show interferon gamma (IFN- $\gamma$ ) or tumor necrosis factor alpha (TNF- $\alpha$ ) secretion, against actin (irrelevant self antigen), reference Spike containing the complete sequence of reference peptides for the Spike protein, B.1.1.7 Spike containing the mutated sequences present in the B.1.1.7 variant that differs from reference (B.1.1.7 Variant on Supplemental Table 7), and B.1.351 Spike containing the mutated sequences present in the B.1.351 variant that differs from reference (B.1.351 Variant on Supplemental Table 7). All T cells were expanded for 10-11 days from peripheral blood mononuclear cells against reference Spike peptides.

\section{REFERENCES}


1 Leung, K., Shum, M. H., Leung, G. M., Lam, T. T. \& Wu, J. T. Early transmissibility assessment of the N501Y mutant strains of SARS-CoV-2 in the United Kingdom, October to November 2020. Euro Surveill 26, doi:10.2807/1560-

7917.ES.2020.26.1.2002106 (2021).

2 Tegally, $\mathrm{H}$. et al. Emergence of a SARS-CoV-2 variant of concern with mutations in spike glycoprotein. Nature, doi:10.1038/s41586-021-03402-9 (2021).

3 CDC. US COVID-19 Cases Caused by Variants, <https://www.cdc.gov/coronavirus/2019-ncov/transmission/variant-cases.html> (2021).

4 Ramanathan, M., Ferguson, I. D., Miao, W. \& Khavari, P. A. SARS-CoV-2 B.1.1.7 and B.1.351 Spike variants bind human ACE2 with increased affinity. bioRxiv, doi:10.1101/2021.02.22.432359 (2021).

5 Wang, P. et al. Antibody Resistance of SARS-CoV-2 Variants B.1.351 and B.1.1.7. Nature, doi:10.1038/s41586-021-03398-2 (2021).

6 Chen, R. E. et al. Resistance of SARS-CoV-2 variants to neutralization by monoclonal and serum-derived polyclonal antibodies. Nat Med, doi:10.1038/s41591-021-01294-w (2021).

7 Jangra, S. et al. The E484K mutation in the SARS-CoV-2 spike protein reduces but does not abolish neutralizing activity of human convalescent and post-vaccination sera. medRxiv, doi:10.1101/2021.01.26.21250543 (2021).

8 Gupta, S., Su, H., Narsai, T. \& Agrawal, S. SARS-CoV-2-Associated T-Cell Responses in the Presence of Humoral Immunodeficiency. Int Arch Allergy Immunol 182, 195-209, doi:10.1159/000514193 (2021).

9 Grifoni, A. et al. Targets of T Cell Responses to SARS-CoV-2 Coronavirus in Humans with COVID-19 Disease and Unexposed Individuals. Cell 181, 1489-1501 e1415, doi:10.1016/j.cell.2020.05.015 (2020). 
10 Rydyznski Moderbacher, C. et al. Antigen-Specific Adaptive Immunity to SARS-CoV-2 in Acute COVID-19 and Associations with Age and Disease Severity. Cell 183, 996-1012 e1019, doi:10.1016/j.cell.2020.09.038 (2020).

11 Collier, D. A. et al. Sensitivity of SARS-CoV-2 B.1.1.7 to mRNA vaccine-elicited antibodies. Nature, doi:10.1038/s41586-021-03412-7 (2021).

12 Diamond, M. et al. SARS-CoV-2 variants show resistance to neutralization by many monoclonal and serum-derived polyclonal antibodies. Res Sq, doi:10.21203/rs.3.rs228079/v1 (2021).

13 Wang, Z. et al. mRNA vaccine-elicited antibodies to SARS-CoV-2 and circulating variants. Nature, doi:10.1038/s41586-021-03324-6 (2021).

14 Tarke, A. et al. Negligible impact of SARS-CoV-2 variants on CD4 (+) and CD8 (+) T cell reactivity in COVID-19 exposed donors and vaccinees. bioRxiv, doi:10.1101/2021.02.27.433180 (2021).

15 Keller, M. D. et al. SARS-CoV-2-specific T cells are rapidly expanded for therapeutic use and target conserved regions of the membrane protein. Blood 136, 2905-2917, doi:10.1182/blood.2020008488 (2020).

16 Jackson, L. A. et al. An mRNA Vaccine against SARS-CoV-2 - Preliminary Report. $N$ Engl J Med 383, 1920-1931, doi:10.1056/NEJMoa2022483 (2020).

17 Tada, T. et al. Neutralization of viruses with European, South African, and United States SARS-CoV-2 variant spike proteins by convalescent sera and BNT162b2 mRNA vaccine-elicited antibodies. bioRxiv, doi:10.1101/2021.02.05.430003 (2021).

18 Burbelo, P. D. et al. Sensitivity in Detection of Antibodies to Nucleocapsid and Spike Proteins of Severe Acute Respiratory Syndrome Coronavirus 2 in Patients With Coronavirus Disease 2019. J Infect Dis 222, 206-213, doi:10.1093/infdis/jiaa273 (2020).

19 Reynisson, B., Alvarez, B., Paul, S., Peters, B. \& Nielsen, M. NetMHCpan-4.1 and NetMHCllpan-4.0: improved predictions of MHC antigen presentation by concurrent 
motif deconvolution and integration of MS MHC eluted ligand data. Nucleic Acids Res 48, W449-W454, doi:10.1093/nar/gkaa379 (2020).

20 Vita, R. et al. The Immune Epitope Database (IEDB): 2018 update. Nucleic Acids Res 47, D339-D343, doi:10.1093/nar/gky1006 (2019). 


\section{Figures}

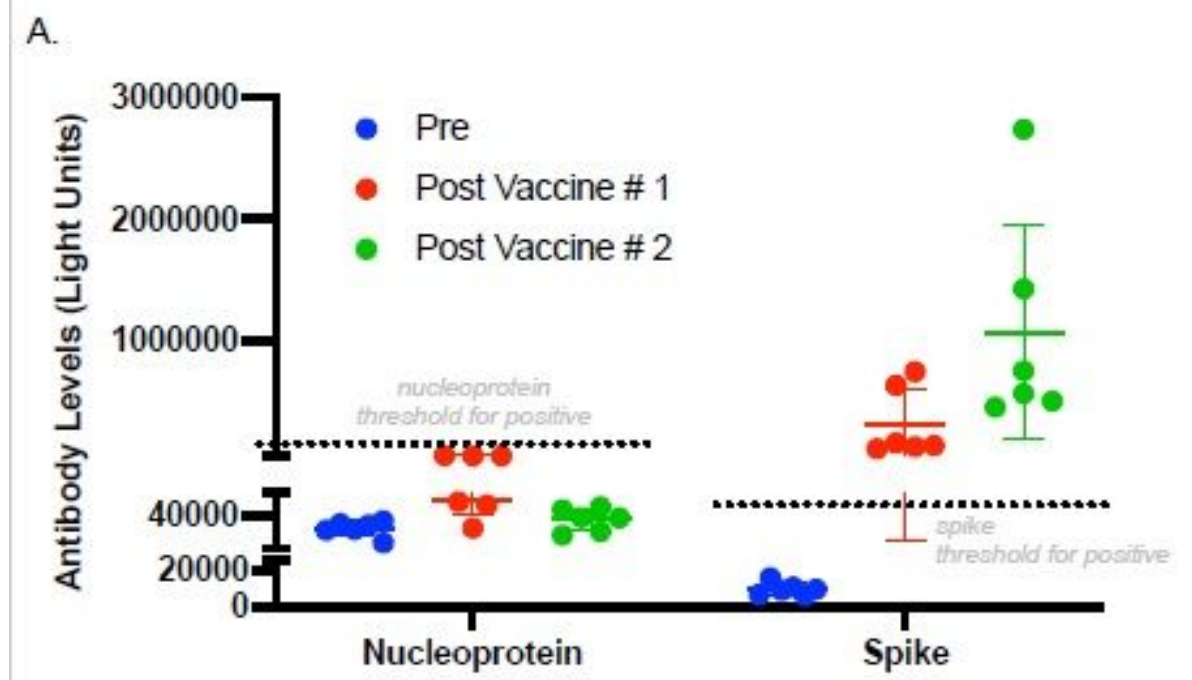

B.

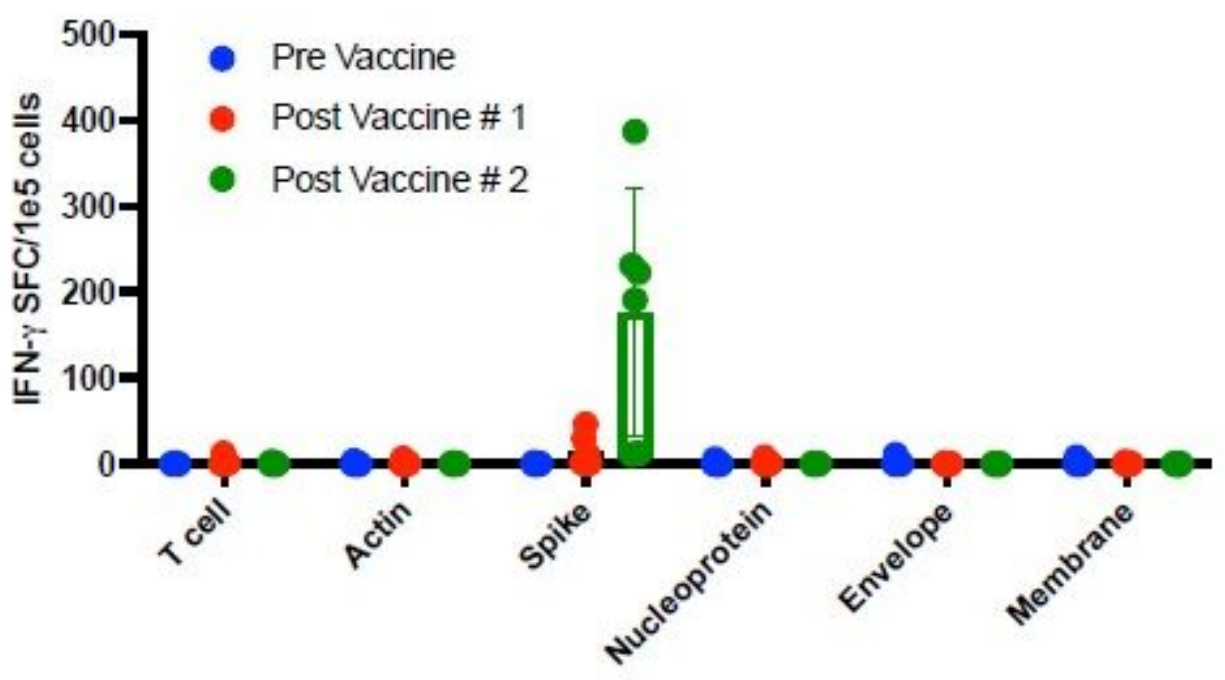

Figure 1

Antibodies and T cells from seronegative donors demonstrate enhanced reactivity to Spike post vaccination. A. Antibody to nucleocapsid and to Spike, measured in six donors immediately before vaccination (blue circles), three weeks after the first vaccine and before administration of the second vaccine (red circles), and three to four weeks after administration of the second vaccine (green circles). B. T cell responses as measured by IFN-y ELISpot after 10-11 day expansion of peripheral blood mononuclear cells with COVID-19 nonstructural antigens Spike, membrane, nucleocapsid, and envelope to zoom in on specific T cells, measured immediately before vaccination (blue circles), three weeks after the first vaccine and before administration of the second vaccine (red circles), and three to four weeks after administration of the second vaccine (green circles). 

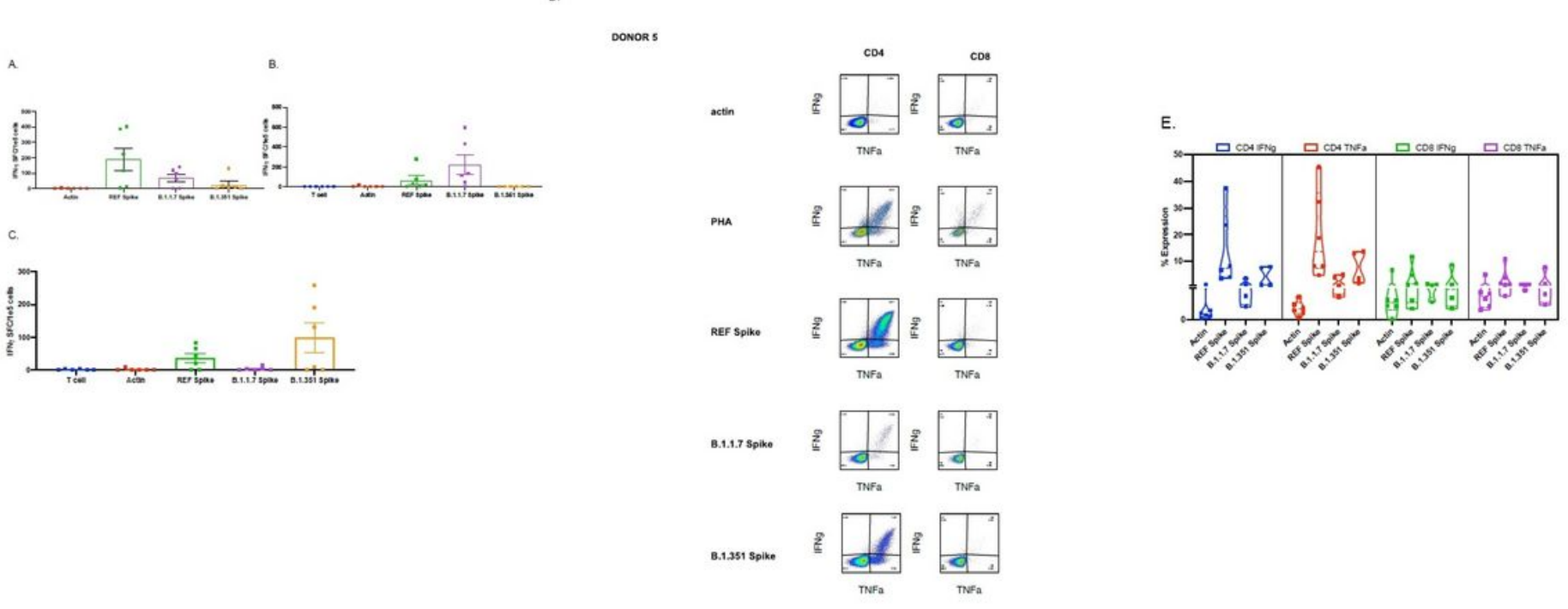

Figure 2

T cells exhibit cross-reactivity against B.1.1.7 and B.1.351 variants. A. T cell responses as measured by IFN- $\gamma$ ELISpot after 10-11 day expansion of peripheral blood mononuclear cells (PBMCs) with COVID-19 reference Spike. Bars depict mean, and error bars show standard deviation. B. T cell responses as measured by IFN-y ELISpot after 10-11 day expansion of peripheral blood mononuclear cells with COVID19 B.1.1.7 Spike variant. Bars depict mean, and error bars show standard deviation. C. T cell responses as measured by IFN-y ELISpot after 10-11 day expansion of peripheral blood mononuclear cells with COVID19 reference Spike. Bars depict mean, and error bars show standard deviation. D. Representative intracellular stain of a donor, showing responses to negative control (actin), to positive control (PHA), and to the three different Spike sequences: reference Spike containing the complete sequence of reference peptides for the Spike protein, B.1.1.7 Spike containing the mutated sequences present in the B.1.1.7 variant that differs from the reference strain, and B.1.351 Spike containing the mutated sequences present in the B.1.351 variant that differs from reference. E. A summary of responses, measured as percent of CD4+ or CD8+ T cells that show interferon gamma (IFN-y) or tumor necrosis factor alpha (TNFa) secretion, against actin (irrelevant self antigen), reference Spike containing the complete sequence of reference peptides for the Spike protein, B.1.1.7 Spike containing the mutated sequences present in the B.1.1.7 variant that differs from reference (B.1.1.7 Variant on Supplemental Table 7), and B.1.351 Spike containing the mutated sequences present in the B.1.351 variant that differs from reference (B.1.351 Variant on Supplemental Table 7). All T cells were expanded for 10-11 days from peripheral blood mononuclear cells against reference Spike peptides.

\section{Supplementary Files}

This is a list of supplementary files associated with this preprint. Click to download. 
- REVISED040821COMPLETESupplementalMaterials.pdf 